Article

\title{
First-Principles Calculation of Transition Metal Hyperfine Coupling Constants with the Strongly Constrained and Appropriately Normed (SCAN) Density Functional and its Hybrid Variants
}

\author{
Dimitrios A. Pantazis \\ Max-Planck-Institut für Kohlenforschung, Kaiser-Wilhelm-Platz, 1, 45470 Mülheim an der Ruhr, Germany; \\ dimitrios.pantazis@kofo.mpg.de; Tel.: +49-208-306-2156
}

Received: 27 October 2019; Accepted: 10 December 2019; Published: 12 December 2019

\begin{abstract}
Density functional theory (DFT) is used extensively for the first-principles calculation of hyperfine coupling constants in both main-group and transition metal systems. As with many other properties, the performance of DFT for hyperfine coupling constants is of variable quality, particularly for transition metal complexes, because it strongly depends on the nature of the chemical system and the type of approximation to the exchange-correlation functional. Recently, a meta-generalized-gradient approximation (mGGA) functional was proposed that obeys all known exact constraints for such a method, known as the Strongly Constrained and Appropriately Normed (SCAN) functional. In view of its theoretically superior formulation a benchmark set of complexes is used to assess the performance of SCAN for the challenging case of transition metal hyperfine coupling constants. In addition, two global hybrid versions of the functional, SCANh and SCAN0, are described and tested. The values computed with the new functionals are compared with experiment and with those of other DFT approximations. Although the original SCAN and the SCAN-based hybrids may offer improved hyperfine coupling constants for specific systems, no uniform improvement is observed. On the contrary, there are specific cases where the new functionals fail badly due to a flawed description of the underlying electronic structure. Therefore, despite these methodological advances, systematically accurate and system-independent prediction of transition metal hyperfine coupling constants with DFT remains an unmet challenge.
\end{abstract}

Keywords: hyperfine coupling; density functional theory; spectroscopy; hybrid functionals

\section{Introduction}

Advancements in the theoretical framework for the calculation of electron paramagnetic resonance (EPR) parameters on the basis of Kohn-Sham density functional theory (DFT) [1], combined with the continuous improvement in the quality of approximate exchange-correlation functionals [2,3], have significantly expanded the scope of DFT calculations beyond simplistic considerations of potential energy surface features, enabling their tight integration with experimental spectroscopy in modern research [4-6]. The ability to compute with reasonable accuracy all properties relevant for EPR spectroscopy, i.e., $g$-values, hyperfine coupling constants, and zero-field splitting parameters, is well established for organic and main-group molecules. On the other hand, it is well documented that the performance of DFT for EPR parameters of transition metal systems is inconsistent and highly system-dependent [4]. Although it is usually possible to identify a DFT-based methodological approach that provides results of useful accuracy for a specific EPR property in a chemically closely related class of transition metal complexes, any given functional thus selected is rarely equally successful when applied to different systems. This limited transferability means that in DFT calculations of EPR 
parameters for transition metal complexes one often relies on fortuitous cancellation of different errors with possibly distinct physical origins and non-transparent methodological causes. Thus, if one is restricted by choice or practical necessity to use DFT instead of rigorous wave function based methods for predicting EPR parameters in transition metal systems, the choice of functional is typically dictated by experience accumulated in previously published studies of related systems or by performing an initial benchmarking study. Among the EPR parameters that react most sensitively to the choice of computational methodology are hyperfine coupling constants [7-14].

Hyperfine coupling refers to the interaction between unpaired electrons and magnetic nuclei, i.e., between the electron spin $\hat{S}$ and the nuclear spin $\hat{I}(\hat{H}=\hat{S} \mathbf{A} \hat{I})$. The hyperfine coupling tensor for a given nucleus $N$ consists of three contributions, the isotropic Fermi contact term $\left(A^{\mathrm{FC}}\right)$, the anisotropic spin-dipolar $\left(A^{\mathrm{SD}}\right)$, and the spin-orbit coupling (SOC) contributions $\left(A^{\mathrm{SO}}\right)$ :

$$
A(N)=A^{\mathrm{FC}}+A^{\mathrm{SD}}+A^{\mathrm{SO}}
$$

The Fermi contact term arises from non-vanishing spin density at the point of the nucleus:

$$
A^{\mathrm{FC}}(N)=\frac{4 \pi}{3} g_{e} g_{N} \beta_{e} \beta_{N}\left\langle S_{z}\right\rangle^{-1} \rho^{\alpha-\beta}\left(\mathbf{R}_{N}\right)
$$

In the above equation $g_{e}$ and $g_{N}$ are the $g$-factors of the electron and the nucleus, and $\beta_{e}$ and $\beta_{N}$ are the electronic and nuclear magnetons. The product $g_{e} g_{N} \beta_{e} \beta_{N}$ is commonly abbreviated as $P_{N}$ and is a scaling factor (with units $\mathrm{MHz} \mathrm{au}^{-3}$ ) fixed for a given nucleus. $\left\langle S_{z}\right\rangle$ is the expectation value of the $\mathrm{z}$ component of the total spin and $\rho^{\alpha-\beta}\left(\mathbf{R}_{N}\right)$ is the spin density at the position of nucleus $N$. The spin dipolar part of the hyperfine tensor describes the magnetic dipole interaction of the magnetic nucleus with the electron magnetic moment:

$$
A_{k l}^{\mathrm{SD}}(N)=\frac{1}{2} P_{N} S_{z}^{-1} \sum_{\mu, v} P_{\mu, v}^{\alpha-\beta}\left\langle\varphi_{\mu}\left|\mathbf{r}_{N}^{-5}\left(\mathbf{r}_{N}^{2} \delta_{k l}-3 \mathbf{r}_{N, k} \mathbf{r}_{N, l}\right)\right| \varphi_{v}\right\rangle
$$

where $\mathbf{r}_{N}=\mathbf{r}-\mathbf{R}_{N}$ and $P_{\mu, v}^{\alpha-\beta}$ is the spin density matrix with the indices denoting basis functions. Finally, spin-orbit coupling gives rise to the second-order contribution to the hyperfine coupling tensor. This can be obtained with the mean-field approximation to the SOC operator (SOMF) [15] as:

$$
A_{k l}^{\mathrm{SO}}(N)=-\frac{1}{S} P_{N} \sum_{\mu, v} \frac{\partial P_{\mu, v}^{\alpha-\beta}}{\partial \hat{\mathrm{I}}_{k}}\left\langle\varphi_{\mu}\left|\hat{z}_{l}^{\mathrm{SOMF}}\right| \varphi_{v}\right\rangle
$$

Practical applications of DFT for the calculation of hyperfine coupling tensors have met with mixed success. For organic radicals usually good results can be obtained with a variety of density functionals in conjunction with basis sets that are developed specifically for the calculation of EPR properties. The picture is different for transition metal systems, where the quality of computed hyperfine coupling tensors varies widely with the nature of the chemical system, the oxidation state of the metal, and the choice of functional. The presence of multiple exchange-coupled transition metal centers introduces additional layers of complication that will not be discussed here [16-18].

The Fermi contact term depends on how a given functional describes core (s orbital) spin polarization induced by unpaired d orbital spin density and, hence, it is sensitive to the quality of the electronic structure description afforded by a particular density functional for a given transition metal complex. Modern functionals typically underestimate core spin polarization, often significantly [7]. Dipolar coupling is principally determined by the spin density distribution in the valence region. Thus it already becomes clear that a functional needs to provide a very balanced description of the core and valence electronic structure and spin density distribution to perform well for both $A^{\mathrm{FC}}$ and $A^{\mathrm{SD}}$. Moreover, the $A^{\mathrm{SO}}$ term can have heightened importance for transition metal complexes compared 
to organic systems, even for first-row transition metal complexes, introducing additional challenges for DFT.

Certain patterns in terms of performance may be identified with respect to the distinct families of density functionals. In the generalized gradient approximation (GGA) to DFT, the density and its gradient are employed. In meta-GGA (mGGA) functionals the Laplacian of the density or the local kinetic energy density are included. Hybrid functionals utilize a GGA or mGGA functional as starting point and incorporate a percentage of exact (Hartree-Fock) exchange, typically empirically adjusted. Double-hybrid functionals incorporate in addition a second-order perturbation term. The underestimation by DFT of core spin polarization can be partly counteracted by admixture of exact exchange in hybrid functionals. However, this is an ad hoc fix that may introduce other problems, for example related to spin contamination, and adversely affect contributions to hyperfine coupling other than the Fermi contact term. Thus, although hybrid functionals are generally considered superior to GGA functionals for specific families of compounds, their use does not automatically guarantee a balanced treatment of the physically diverse contributions to the hyperfine coupling tensor and hence cannot achieve uniformly higher accuracy. Local hybrids with variable position-dependent admixture of exact exchange are being developed as a potential way forward [13]. Nevertheless, it is important to seek improvements in the theoretical method already at the GGA and mGGA levels so that reliance on error cancellation by selective inclusion of exact exchange is minimized or altogether avoided.

The development of new density functionals is being pursued along conceptually distinct approaches [19-21]. One of them adopts the empirical adjustment of several parameters against datasets so that a functional reproduces as well as possible known values for a series of reference systems. Typically, the reference values are various types of energetics; spectroscopic parameters are seldom, if ever, used to guide the optimization of the empirical parameters. An alternative approach emphasizes the necessity of obeying known constraints and physical conditions (such as various upper/lower bounds, exact limiting cases and scaling relationships) on the assumption that a functional which is able by construction to obey as many as possible of these constraints and is able to converge to the correct answers for limiting cases should in principle be describing the actual physics of a system in a fundamentally superior manner. This in turn leads to the expectation of improved results for all kinds of properties. One of the latest functionals to be constructed along the lines of this non-empirical approach is the strongly constrained and appropriately normed (SCAN) functional by Sun, Ruzsinszky, and Perdew [22]. SCAN is an mGGA functional and the first to obey all known exact constraints (17 in total) that can apply to a density functional of this type. In addition, it is either exact or nearly exact for a set of "appropriate norms" that include rare-gas atoms and non-bonded interactions [22].

Studies that examined the performance of SCAN and certain derivative functionals have already indicated that it performs well for a variety of energy-related properties, usually surpassing previous GGA and mGGA approaches [22-31]. However, it is unclear whether these advances also translate into improved description of the physical mechanisms that give rise to the distinct contributions to hyperfine coupling. In the present study the performance of SCAN is tested for the challenging case of transition metal hyperfine coupling tensors using a reference set of transition metal complexes that cover most of the $3 \mathrm{~d}$ elements. Moreover, two hybrid versions of the functional that incorporate either $10 \%$ or $25 \%$ of Hartree-Fock exchange (SCANh and SCAN0) are defined and assessed. The results are compared to experiment as well as to representative GGA, meta-GGA, and hybrid-meta-GGA functionals. In addition, a series of methodological considerations regarding the applicability of SCAN are examined.

\section{Results}

\subsection{Reference Set of Transition Metal Systems}

For the purposes of the present study a set of small first-row transition metal containing molecules is used. The compounds almost cover the complete $3 \mathrm{~d}$ series and are selected because they have 
experimentally known and resolved hyperfine coupling constants and have been employed previously in analogous evaluations of theoretical methods $[10,32]$. The compounds used along with their total spin states are: $\mathrm{ScO}(S=1 / 2), \mathrm{TiF}_{3}(S=1 / 2),\left[\mathrm{V}\left(\mathrm{H}_{2} \mathrm{O}\right)_{6}\right]^{2+}(S=3 / 2),\left[\mathrm{VO}\left(\mathrm{H}_{2} \mathrm{O}\right)_{5}\right]^{2+}(S=1 / 2), \operatorname{MnF}(S=2)$, $\mathrm{MnO}_{3}(S=1 / 2),\left[\mathrm{Mn}\left(\mathrm{H}_{2} \mathrm{O}\right)_{6}\right]^{2+}(S=5 / 2), \mathrm{Mn}(\mathrm{CO})_{5}(S=1 / 2),\left[\mathrm{Fe}(\mathrm{CO})_{5}\right]^{+}(S=1 / 2), \mathrm{NiH}(\mathrm{CO})_{3}(S=1 / 2)$, $\left[\mathrm{Ni}(\mathrm{mnt})_{2}\right]^{-}(S=1 / 2),\left[\mathrm{Cu}\left(\mathrm{NH}_{3}\right)_{4}\right]^{2+}(S=1 / 2),\left[\mathrm{Cu}(\mathrm{dtc})_{2}\right](S=1 / 2)$, and $\left[\mathrm{Cu}(\mathrm{en})_{2}\right]^{2+}(S=1 / 2)$, where mnt $=$ maleonitriledithiolate, $\mathrm{dtc}=$ dithiocarbamate, and en $=$ ethylenediamine. For completeness, it is noted that the isotopes and nuclear spins assumed here are: ${ }^{45} \mathrm{Sc}(I=7 / 2),{ }^{47} \mathrm{Ti}(I=5 / 2),{ }^{51} \mathrm{~V}(I=7 / 2)$, ${ }^{55} \mathrm{Mn}(I=5 / 2),{ }^{57} \mathrm{Fe}(I=1 / 2),{ }^{61} \mathrm{Ni}(I=3 / 2)$, and ${ }^{63} \mathrm{Cu}(I=3 / 2)$. The default values implemented in the ORCA program package [33] were employed for the scaled nuclear $g$-values $P_{N}$.

\subsection{Performance of SCAN in Comparison With Other Density Functionals}

To place the performance of SCAN in context, Table 1 compares the detailed hyperfine parameters obtained by SCAN with those obtained using standard density functionals, the GGA BP86 [34,35], the mGGA TPSS [36], its hybrid version TPSSh (10\% exact exchange) [37], B3LYP (20\% exact exchange) [38,39], BHandHLYP (50\% exact exchange), and CAM-B3LYP (range-dependent exact exchange from $19 \%$ to $65 \%$ ) [40]. The table includes results from hybrid versions of SCAN that will be discussed in more detail in the following section. In all cases appropriate core-property $\mathrm{CP}(\mathrm{PPP})$ basis sets [41] with extensive polarization and a highly flexible inner-core subset of s-type functions were used (see Computational Details). The table includes experimental values for the hyperfine coupling constants. In some cases, experimental values are also available for the distinct contributions to the hyperfine tensor $\left(A^{\mathrm{FC}}\right.$ and $\left.A^{\mathrm{SD}}\right)$ and these are also listed in the respective columns. It is noted that the HFC components are listed in all cases from smaller to larger absolute value. The computed total values reported in Table 1 incorporate the spin-orbit coupling contributions $\left(A^{\mathrm{SO}}\right)$, which are only significant for $\mathrm{Cu}$ and secondarily for $\mathrm{Ni}$ complexes. The $A^{\mathrm{SO}}$ values are listed for all compounds and functionals in Table A1 of Appendix A.

Table 1. Hyperfine coupling constants (in $\mathrm{MHz}$ ) computed with different density functionals.

\begin{tabular}{ccccccccc}
\hline Compound & Functional & $\boldsymbol{A}_{\mathbf{1 1}}$ & $\boldsymbol{A}_{\mathbf{2 2}}$ & $\boldsymbol{A}_{\mathbf{3 3}}$ & $\boldsymbol{A}^{\text {FC }}$ & $\boldsymbol{A}_{\mathbf{1 1}}$ SD & $\boldsymbol{A}_{\mathbf{2 2}}{ }^{\text {SD }}$ & $\boldsymbol{A}_{\mathbf{3 3}}$ SD \\
\hline \multirow{2}{*}{ ScO } & BP86 & 1923.2 & 1923.2 & 1981.8 & 1943.3 & -19.3 & -19.3 & 38.6 \\
& TPSS & 1766.1 & 1766.1 & 1825.7 & 1786.5 & -19.6 & -19.6 & 39.3 \\
& TPSSh & 1763.5 & 1763.5 & 1825.1 & 1784.6 & -20.3 & -20.3 & 40.6 \\
& B3LYP & 1970.0 & 1970.0 & 2031.2 & 1991.0 & -20.1 & -20.1 & 40.2 \\
& BHandHLYP & 1932.1 & 1932.1 & 2000.5 & 1955.6 & -22.5 & -22.5 & 45.1 \\
& CAM-B3LYP & 2024.0 & 2024.0 & 2087.2 & 2045.6 & -20.8 & -20.8 & 41.7 \\
& SCAN & -218.3 & -218.3 & -328.3 & -252.1 & 35.2 & 35.2 & -70.5 \\
& SCANh & -230.1 & -230.1 & -342.2 & -264.5 & 35.8 & 35.8 & -71.7 \\
& SCAN0 & -247.7 & -247.7 & -363.2 & -283.2 & 36.8 & 36.8 & -73.6 \\
& Exp. [42] & 1922 & 1922 & 1997 & 1947 & -25 & -25 & 50 \\
\hline \multirow{2}{*}{ SiF $_{3}$} & BP86 & -210.9 & -210.9 & -241.6 & -223.1 & 9.4 & 9.4 & -18.7 \\
& TPSS & -195.8 & -195.8 & -224.2 & -206.9 & 8.8 & 8.8 & -17.6 \\
& TPSSh & -181.1 & -181.1 & -210.2 & -192.6 & 8.9 & 8.9 & -17.9 \\
& B3LYP & -183.7 & -183.7 & -217.8 & -197.5 & 10.2 & 10.2 & -20.4 \\
& BHandHLYP & -143.1 & -143.1 & -178.5 & -158.3 & 10.1 & 10.1 & -20.3 \\
& CAM-B3LYP & -175.7 & -175.7 & -208.0 & -187.2 & 10.5 & 10.5 & -20.9 \\
& SCAN & 34.0 & 34.1 & 84.1 & 48.1 & -16.2 & -14.9 & 31.1 \\
& SCANh & 34.9 & 35.2 & 86.4 & 49.4 & -15.3 & -16.4 & 31.7 \\
& SCAN0 & 36.0 & 37.0 & 89.9 & 51.1 & -15.9 & -16.7 & 32.6 \\
& Exp. [43] & -178 & -178 & -199 & -185 & 7 & 7 & -14 \\
\hline
\end{tabular}


Table 1. Cont.

\begin{tabular}{|c|c|c|c|c|c|c|c|c|}
\hline Compound & Functional & $A_{11}$ & $A_{22}$ & $A_{33}$ & $A^{\mathrm{FC}}$ & $A_{11} \mathrm{SD}$ & $A_{22} \mathrm{SD}$ & $A_{33} \mathrm{SD}$ \\
\hline \multirow[t]{10}{*}[\mathrm{V}(\mathrm{H}_{2}\mathrm{O})_{6}]{$^{2+}$} & BP86 & -170.5 & -170.5 & -170.8 & -161.2 & 0.0 & 0.0 & -0.1 \\
\hline & TPSS & -179.8 & -179.8 & -180.0 & -171.6 & 0.0 & 0.0 & -0.1 \\
\hline & TPSSh & -191.9 & -191.9 & -192.1 & -183.4 & 0.0 & 0.0 & -0.1 \\
\hline & B3LYP & -169.4 & -169.4 & -169.7 & -159.4 & 0.1 & 0.1 & -0.1 \\
\hline & BHandHLYP & -202.2 & -202.2 & -202.6 & -191.4 & 0.1 & 0.1 & -0.1 \\
\hline & CAM-B3LYP & -162.1 & -162.1 & -162.3 & -158.1 & 0.0 & 0.0 & -0.1 \\
\hline & SCAN & -230.4 & -230.4 & -230.7 & -223.4 & 0.1 & 0.1 & -0.2 \\
\hline & SCANh & -236.7 & -236.7 & -237.1 & -229.5 & 0.1 & 0.1 & -0.2 \\
\hline & SCAN0 & -245.1 & -245.1 & -245.4 & -237.4 & 0.1 & 0.1 & -0.2 \\
\hline & Exp. [44] & -247 & -247 & -247 & & & & \\
\hline \multirow{10}{*}[\mathrm{VO}(\mathrm{H}_{2}\mathrm{O})_{5}]{$^{2+}$} & BP86 & -162.4 & -163.3 & -471.0 & -252.3 & 97.1 & 96.4 & -193.5 \\
\hline & TPSS & -179.8 & -180.5 & -485.8 & -270.4 & 97.2 & 96.7 & -193.9 \\
\hline & TPSSh & -204.5 & -205.0 & -514.5 & -295.5 & 98.1 & 97.8 & -195.9 \\
\hline & B3LYP & -178.8 & -179.1 & -503.2 & -271.8 & 101.2 & 101.0 & -202.1 \\
\hline & BHandHLYP & -269.2 & -272.8 & -599.0 & -361.4 & 102.7 & 99.5 & -202.2 \\
\hline & CAM-B3LYP & -175.4 & -175.9 & -489.0 & -273.6 & 101.7 & 101.2 & -202.9 \\
\hline & SCAN & -215.6 & -216.0 & -500.4 & -299.3 & 90.9 & 90.4 & -181.2 \\
\hline & SCANh & -238.9 & -239.6 & -527.1 & -323.1 & 91.8 & 91.1 & -183.0 \\
\hline & SCANO & -275.4 & -276.7 & -568.0 & -360.0 & 93.0 & 91.7 & -184.7 \\
\hline & Exp. [45] & -208 & -208 & -547 & & & & \\
\hline \multirow[t]{10}{*}{$\mathrm{MnF}$} & BP86 & 433.5 & 433.5 & 495.1 & 444.3 & -14.7 & -14.7 & 29.3 \\
\hline & TPSS & 413.8 & 413.8 & 478.8 & 432.7 & -16.1 & -16.1 & 32.1 \\
\hline & TPSSh & 397.3 & 397.3 & 467.2 & 417.0 & -17.3 & -17.3 & 34.5 \\
\hline & B3LYP & 443.0 & 443.0 & 507.1 & 441.8 & -16.5 & -16.5 & 32.9 \\
\hline & BHandHLYP & 457.2 & 457.2 & 525.3 & 430.7 & -18.7 & -18.7 & 37.4 \\
\hline & CAM-B3LYP & 402.3 & 402.3 & 464.2 & 423.7 & -17.9 & -17.9 & 35.7 \\
\hline & SCAN & 492.4 & 492.4 & 547.4 & 511.2 & -13.7 & -13.7 & 27.5 \\
\hline & SCANh & 478.7 & 478.7 & 537.9 & 498.5 & -14.8 & -14.7 & 29.5 \\
\hline & SCANO & 459.9 & 459.9 & 525.5 & 480.8 & -16.1 & -16.1 & 32.3 \\
\hline & Exp. [46] & 430 & 430 & 466 & 442 & -12 & -12 & 24 \\
\hline \multirow[t]{10}{*}{$\mathrm{MnO}_{3}$} & BP86 & 1818.4 & 1818.4 & 2124.9 & 1934.2 & -96.9 & -96.9 & 193.7 \\
\hline & TPSS & 1750.4 & 1750.4 & 2040.1 & 1859.4 & -92.0 & -91.9 & 183.9 \\
\hline & TPSSh & 1590.3 & 1590.4 & 1916.6 & 1712.5 & -104.4 & -104.4 & 208.8 \\
\hline & B3LYP & 1520.0 & 1520.0 & 1917.6 & 1667.8 & -128.5 & -128.4 & 256.9 \\
\hline & BHandHLYP & 1366.2 & 1366.2 & 1607.1 & 1455.8 & -75.9 & -75.9 & 151.9 \\
\hline & CAM-B3LYP & 1427.9 & 1427.9 & 1874.9 & 1586.5 & -147.2 & -147.2 & 294.4 \\
\hline & SCAN & 1714.2 & 1714.3 & 2016.3 & 1828.5 & -97.2 & -97.1 & 194.4 \\
\hline & SCANh & 1502.8 & 1502.9 & 1877.0 & 1642.9 & -121.8 & -121.7 & 243.5 \\
\hline & SCANO & 1163.6 & 1164.1 & 1656.6 & 1346.2 & -162.8 & -162.3 & 325.1 \\
\hline & Exp. [47] & 1532 & 1532 & 1775 & 1613 & -81 & -81 & 162 \\
\hline \multirow[t]{10}{*}[\mathrm{Mn}(\mathrm{H}_{2}\mathrm{O})_{6}]{$^{2+}$} & BP86 & -162.0 & -162.2 & -162.2 & -159.2 & 0.1 & 0.0 & 0.0 \\
\hline & TPSS & -173.2 & -173.4 & -173.4 & -170.4 & 0.1 & -0.1 & -0.1 \\
\hline & TPSSh & -187.7 & -188.0 & -188.0 & -185.1 & 0.2 & -0.1 & -0.1 \\
\hline & B3LYP & -163.7 & -163.9 & -163.9 & -161.3 & 0.1 & -0.1 & -0.1 \\
\hline & BHandHLYP & -197.4 & -197.7 & -197.7 & -195.5 & 0.2 & -0.1 & -0.1 \\
\hline & CAM-B3LYP & -165.1 & -165.3 & -165.3 & -162.9 & 0.1 & -0.1 & -0.1 \\
\hline & SCAN & -221.7 & -222.1 & -222.1 & -219.0 & 0.2 & -0.1 & -0.1 \\
\hline & SCANh & -229.7 & -230.1 & -230.1 & -227.1 & 0.3 & -0.1 & -0.1 \\
\hline & SCANO & -240.3 & -240.7 & -240.7 & -238.0 & 0.3 & -0.1 & -0.1 \\
\hline & Exp. [48] & -245 & -245 & -245 & & & & \\
\hline
\end{tabular}


Table 1. Cont.

\begin{tabular}{|c|c|c|c|c|c|c|c|c|}
\hline Compound & Functional & $A_{11}$ & $A_{22}$ & $A_{33}$ & $A^{\mathrm{FC}}$ & $A_{11} \mathrm{SD}$ & $A_{22} \mathrm{SD}$ & $A_{33} \mathrm{SD}$ \\
\hline \multirow[t]{10}{*}{$\mathrm{Mn}(\mathrm{CO})_{5}$} & BP86 & -80.9 & -81.0 & 202.5 & 12.0 & -95.9 & -96.0 & 191.8 \\
\hline & TPSS & -79.2 & -79.3 & 195.9 & 11.6 & -92.9 & -93.0 & 185.9 \\
\hline & TPSSh & -83.4 & -83.5 & 190.7 & 7.2 & -92.7 & -92.8 & 185.5 \\
\hline & B3LYP & -81.7 & -81.8 & 201.7 & 11.1 & -96.3 & -96.4 & 192.7 \\
\hline & BHandHLYP & -74.2 & -74.3 & 176.8 & 9.9 & -85.7 & -85.8 & 171.5 \\
\hline & CAM-B3LYP & -83.1 & -83.2 & 207.9 & 15.5 & -96.8 & -96.9 & 193.7 \\
\hline & SCAN & -109.1 & -109.2 & 161.0 & -19.4 & -90.7 & -90.8 & 181.5 \\
\hline & SCANh & -106.4 & -106.5 & 160.9 & -17.5 & -89.8 & -89.9 & 179.7 \\
\hline & SCANO & -96.2 & -96.4 & 162.5 & -9.9 & -87.0 & -87.2 & 174.2 \\
\hline & Exp. [49] & -86 & -86 & 190 & 6 & -92 & -2 & 194 \\
\hline \multirow[t]{10}{*}[\mathrm{Fe}(\mathrm{CO})_{5}]{$^{+}$} & BP86 & -15.2 & -15.3 & 36.5 & 0.6 & -18.1 & -18.1 & 36.1 \\
\hline & TPSS & -14.9 & -14.9 & 36.1 & 1.0 & -17.7 & -17.7 & 35.5 \\
\hline & TPSSh & -16.9 & -17.0 & 34.9 & -1.0 & -18.1 & -18.2 & 36.3 \\
\hline & B3LYP & -17.4 & -17.5 & 36.4 & -1.5 & -19.2 & -19.2 & 38.4 \\
\hline & BHandHLYP & -21.5 & -21.5 & 32.2 & -6.3 & -19.7 & -19.8 & 39.5 \\
\hline & CAM-B3LYP & -20.8 & -20.9 & 37.2 & -1.7 & -19.6 & -19.6 & 39.2 \\
\hline & SCAN & -19.5 & -19.5 & 31.7 & -3.1 & -17.5 & -17.6 & 35.1 \\
\hline & SCANh & -21.3 & -21.3 & 30.4 & -4.8 & -17.8 & -17.8 & 35.6 \\
\hline & SCANO & -23.7 & -23.8 & 28.5 & -7.3 & -18.2 & -18.2 & 36.4 \\
\hline & Exp. [50] & -17 & -17 & 28 & -2 & -15 & -15 & 30 \\
\hline \multirow[t]{10}{*}{$\mathrm{NiH}(\mathrm{CO})_{3}$} & BP86 & 50.7 & 50.7 & -88.1 & 12.8 & 50.1 & 50.2 & -100.3 \\
\hline & TPSS & 49.5 & 49.6 & -91.3 & 11.0 & 50.8 & 50.8 & -101.5 \\
\hline & TPSSh & 52.6 & 52.6 & -94.1 & 15.2 & 53.9 & 54.0 & -107.9 \\
\hline & B3LYP & 55.3 & 55.3 & -95.0 & 21.7 & 57.2 & 57.2 & -114.3 \\
\hline & BHandHLYP & 46.9 & 46.9 & -107.7 & 36.1 & 67.6 & 67.6 & -135.2 \\
\hline & CAM-B3LYP & 77.4 & 77.5 & -95.5 & 25.1 & 59.8 & 59.8 & -119.7 \\
\hline & SCAN & 59.8 & 59.9 & -91.7 & 17.2 & 53.9 & 53.9 & -107.8 \\
\hline & SCANh & 69.0 & 69.1 & -88.3 & 27.2 & 56.7 & 56.8 & -113.5 \\
\hline & SCANO & 81.4 & 81.5 & -83.3 & 43.0 & 61.0 & 61.0 & -122.0 \\
\hline & Exp. [51] & 53 & 53 & -79 & & & & \\
\hline \multirow[t]{10}{*}[\mathrm{Ni}(\mathrm{mnt})_{2}]{$^{-}$} & BP86 & -9.4 & -11.1 & 47.7 & 24.7 & -30.1 & -25.7 & 55.8 \\
\hline & TPSS & -16.3 & -17.2 & 45.3 & 19.5 & -31.8 & -26.4 & 58.2 \\
\hline & TPSSh & -16.6 & -17.3 & 45.7 & 23.4 & -26.4 & -34.8 & 61.2 \\
\hline & B3LYP & -13.8 & -16.0 & 41.1 & 26.8 & -24.6 & -33.6 & 58.2 \\
\hline & BHandHLYP & 6.3 & -13.5 & 35.2 & 23.9 & -12.4 & -15.4 & 27.8 \\
\hline & CAM-B3LYP & -0.9 & -11.1 & 75.8 & 28.0 & -24.7 & -36.1 & 60.8 \\
\hline & SCAN & 1.1 & 1.8 & 70.1 & 38.9 & -27.9 & -32.9 & 60.8 \\
\hline & SCANh & 0.1 & -2.4 & 69.4 & 40.4 & -27.5 & -36.7 & 64.3 \\
\hline & SCANO & 1.8 & -8.6 & 64.8 & 43.9 & -24.3 & -41.1 & 65.4 \\
\hline & Exp. [52] & $<6$ & 9 & 45 & & & & \\
\hline \multirow{10}{*}[\mathrm{Cu}(\mathrm{NH}_{3})_{4}]{$^{2+}$} & BP86 & -11.1 & -11.1 & -516.4 & -264.4 & 206.2 & 206.2 & -412.5 \\
\hline & TPSS & -6.9 & -7.0 & -542.6 & -266.0 & 213.5 & 213.4 & -426.9 \\
\hline & TPSSh & -25.7 & -25.7 & -593.7 & -308.7 & 229.9 & 229.8 & -459.7 \\
\hline & B3LYP & -18.6 & -18.6 & -584.0 & -324.1 & 240.7 & 240.7 & -481.4 \\
\hline & BHandHLYP & -2.7 & -2.8 & -618.4 & -388.1 & 288.1 & 288.0 & -576.1 \\
\hline & CAM-B3LYP & -67.4 & -67.4 & -755.9 & -342.0 & 250.8 & 250.7 & -501.4 \\
\hline & SCAN & -48.8 & -50.5 & -684.6 & -326.4 & 239.7 & 238.0 & -477.7 \\
\hline & SCANh & -66.0 & -67.8 & -734.3 & -363.0 & 254.1 & 252.2 & -506.3 \\
\hline & SCANO & -76.6 & -77.8 & -782.4 & -401.4 & 273.2 & 271.8 & -545.0 \\
\hline & Exp. [53] & -27 & -27 & -586 & & & & \\
\hline
\end{tabular}


Table 1. Cont.

\begin{tabular}{ccccccccc}
\hline Compound & Functional & $\boldsymbol{A}_{\mathbf{1 1}}$ & $\boldsymbol{A}_{\mathbf{2 2}}$ & $\boldsymbol{A}_{\mathbf{3 3}}$ & $A^{\mathrm{FC}}$ & $\boldsymbol{A}_{\mathbf{1 1}}$ SD & $\boldsymbol{A}_{\mathbf{2 2}}{ }^{\text {SD }}$ & $\boldsymbol{A}_{\mathbf{3 3}}$ SD \\
\hline$\left[\mathrm{Cu}(\mathrm{dtc})_{2}\right]$ & BP86 & -45.5 & -50.0 & -393.6 & -199.2 & 132.9 & 129.8 & -262.7 \\
& TPSS & -46.6 & -50.5 & -413.9 & -207.7 & 139.4 & 136.7 & -276.1 \\
& TPSSh & -74.3 & -78.7 & -468.2 & -251.1 & 151.5 & 148.3 & -299.8 \\
& B3LYP & -78.3 & -83.8 & -479.8 & -264.5 & 157.9 & 154.1 & -312.0 \\
& BHandHLYP & -111.8 & -116.5 & -627.2 & -376.4 & 214.7 & 211.1 & -425.7 \\
& CAM-B3LYP & -133.6 & -139.4 & -606.1 & -319.2 & 171.1 & 165.9 & -337.0 \\
& SCAN & -97.5 & -100.6 & -510.5 & -271.7 & 153.3 & 151.1 & -304.4 \\
& SCANh & -116.5 & -120.2 & -556.7 & -305.9 & 165.2 & 162.5 & -327.7 \\
& SCAN0 & -137.3 & -141.4 & -624.4 & -353.6 & 186.0 & 182.9 & -368.9 \\
& Exp. [54] & -107 & -125 & -476 & & & & \\
\hline$\left[\mathrm{Cu}(\mathrm{en})_{2}\right]^{2+}{ }^{2+}$ & BP86 & -43.4 & -43.5 & -536.3 & -279.3 & 197.3 & 196.2 & -393.5 \\
& TPSS & -41.5 & -41.6 & -560.6 & -283.8 & 204.3 & 203.2 & -407.5 \\
& TPSSh & -66.2 & -66.5 & -617.6 & -330.9 & 220.4 & 218.9 & -439.3 \\
& B3LYP & -64.5 & -64.8 & -619.4 & -348.7 & 231.2 & 229.7 & -460.9 \\
& BHAndHLYP & -58.8 & -58.9 & -681.9 & -423.4 & 282.4 & 281.6 & -564.1 \\
& CAM-B3LYP & -105.3 & -107.1 & -773.3 & -370.3 & 242.9 & 240.7 & -483.6 \\
& SCAN & -91.1 & -91.9 & -695.1 & -351.9 & 226.9 & 225.8 & -452.7 \\
& SCANh & -113.5 & -113.9 & -752.2 & -393.9 & 241.9 & 240.9 & -482.9 \\
& SCAN0 & -128.9 & -130.0 & -13.8 & -439.4 & 263.8 & 262.6 & -526.4
\end{tabular}

The picture emerging from the data in Table 1 is largely congruent with established experience from spectroscopy-oriented DFT applications in the sense that no single functional is a clearly superior performer across the board. TPSSh provides results closest to experiment for several compounds, SCAN or one of its hybrids for others, while BP86 also performs well in specific cases. There is no example for which TPSS is the best performing functional. The performance of the closely related functionals B3LYP, BHandHLYP and CAM-B3LYP, which differ in the percentage of global or range-dependent exact exchange, is not sufficiently systematic to allow for specific trends to be established. Overall, the variation in results and functional performance is so large that we prefer to refrain from reporting average errors. Such metrics cannot accurately reflect the deviations in individual components of the hyperfine and would be dominated by outliers, and therefore are not considered reliable in guiding method selection for properties where the performance of DFT functionals is unsystematic.

Going through the list of molecules one can see that BP86 is the "best" functional for ScO. TPSS and TPSSh both underestimate the magnitude of the hyperfine coupling constants, principally because they underestimate the Fermi contact term $A^{\mathrm{FC}}$. Interestingly, both functionals yield almost identical values, suggesting that the exact exchange included in the hybrid TPSSh has absolutely no effect on core spin polarization. This is consistent with the fact that the sequence of B3LYP, BHandHLYP, and CAM-B3LYP hybrids perform similarly despite their fundamentally different exact exchange admixture. However, the most surprising result for this molecule concerns the SCAN functional, which diverges drastically from the other functionals and fails spectacularly to approximate the experimental values even qualitatively. If only the Fermi contact term would be different, then this problem could be attributed to a flawed description of core spin polarization. However, the spin-dipolar parameters are also qualitatively different from other methods, indicating that the origin of the problem is in the description of the valence electronic configuration itself. Indeed, by inspecting in detail the electronic structure of $\mathrm{ScO}$ as obtained from the different functionals, it is confirmed that whereas all other functionals place the sole unpaired electron in an s valence orbital, SCAN converges to a solution where the unpaired electron resides instead in a d orbital $\left(3 \mathrm{~d}_{x y}\right)$. It was confirmed with additional calculations that this is not a technical issue of converging to a local minimum during the self-consistent field procedure, but it is indeed intrinsically how SCAN describes this molecule. 
Therefore, the divergence of this method for $\mathrm{ScO}$ is due to the fundamentally different description of the ground-state electronic structure.

A similar type of problem is encountered in the next compound of the test set, $\mathrm{TiF}_{3}$. In this case CAM-B3LYP and TPSSh yield hyperfine coupling values closest to experiment. In contrast to all other functionals that describe the spin density as distributed in a hybrid sd orbital with predominant $\mathrm{d}$ character, SCAN yields a solution where the electron resides entirely in a pure $d_{z^{2}}$ orbital of Ti with no $\mathrm{s}$ orbital contribution. Thus, for both $\mathrm{ScO}$ and $\mathrm{TiF}_{3} \mathrm{SCAN}$ fails simply due to the description of the valence region, something that comes across also from the fact that the $A^{\mathrm{SD}}$ values from SCAN are qualitatively different from those of all other functionals. This behavior hints at a potentially more general problem of the method, which would require closer scrutiny in dedicated future studies of transition metal systems.

In a way that is representative of the notorious unpredictability of DFT, SCAN, and its hybrid variants provide good hyperfine coupling constants for the next two compounds, $\left[\mathrm{V}\left(\mathrm{H}_{2} \mathrm{O}\right)_{6}\right]^{2+}$ and $\left.\mathrm{VO}\left(\mathrm{H}_{2} \mathrm{O}\right)_{5}\right]^{2+}$, with TPSSh being the second-best. The success of SCAN for $\left[\mathrm{V}\left(\mathrm{H}_{2} \mathrm{O}\right)_{6}\right]^{2+}$ appears to be in the description of core polarization because of the larger $A^{\mathrm{FC}}$ term. However, in the anisotropic vanadyl complex $\left[\mathrm{VO}\left(\mathrm{H}_{2} \mathrm{O}\right)_{5}\right]^{2+}$ the role of $A^{\mathrm{SD}}$ also becomes important and in this case the divergence of SCAN from the other functionals affects the total values in a negative way as the component of largest magnitude is suppressed compared to TPSSh.

${ }^{55} \mathrm{Mn}$ hyperfine coupling constants are widely employed for probing the electronic structure of inorganic systems and biological cofactors, so they have received extensive attention in the experimental and computational literature [16-18,56-68]. The performance of different functionals for the four small Mn compounds of the present set highlights why careful benchmarking on a series of closely related complexes is always required for meaningful applications of DFT in this field. Focusing only on the values of the hyperfine coupling constants and how they compare with experiment, the best performing functional turns out to be BP86 for MnF, TPSSh, and B3LYP for $\mathrm{MnO}_{3}$, SCAN (and even more so, SCAN0) for $\left[\mathrm{Mn}\left(\mathrm{H}_{2} \mathrm{O}\right)_{6}\right]^{2+}$, and TPSSh again for $\mathrm{Mn}(\mathrm{CO})_{5}$. In no case can the results be considered very good; the hyperfine coupling values in the more anisotropic systems reveal starkly the importance of getting right both the $A^{\mathrm{FC}}$ and the $A^{\mathrm{SD}}$ terms, which none of the functionals appears to achieve reliably. Interestingly, SCAN-based functionals are again the best performing methods for a hexa-aquo complex, similarly to the vanadium case. For the carbonyl compound $\mathrm{Mn}(\mathrm{CO})_{5}$ SCAN produces an $A^{\mathrm{FC}}$ term with inverted sign, i.e., opposite spin polarization compared to the other functionals, which results in deviating total values for the hyperfine coupling despite the fact that the $A^{\mathrm{SD}}$ terms are rather similar for all methods.

The next two molecules, $\left[\mathrm{Fe}(\mathrm{CO})_{5}\right]^{+}$and $\mathrm{NiH}(\mathrm{CO})_{3}$, are examples where all methods are reasonably well-behaved and do not produce widely varying values in absolute terms for the hyperfine coupling constants. The picture is somewhat different for $\left[\mathrm{Ni}(\mathrm{mnt})_{2}\right]^{-}$. Here SCAN deviates more strongly from the other methods, even though there are no qualitative differences in the description of the electronic structure.

Finally, it proves difficult yet again to single out a specific density functional for the three copper complexes $\left[\mathrm{Cu}\left(\mathrm{NH}_{3}\right)_{4}\right]^{2+},\left[\mathrm{Cu}(\mathrm{dtc})_{2}\right]$, and $\left[\mathrm{Cu}(\mathrm{en})_{2}\right]^{2+}$, since the experimental values are approximated with different degrees of success by different methods in each case. The difficulty of the problem is compounded in the case of copper by the fact that $A^{\mathrm{SO}}$ terms become comparable in magnitude to the other components of the hyperfine interaction (Table A1). It is noteworthy that SCAN deviates significantly from experiment and from the best performing TPSSh functional in the case of $\left[\mathrm{Cu}\left(\mathrm{NH}_{3}\right)_{4}\right]^{2+}$, a simple system often used for method calibration purposes. For $\left[\mathrm{Cu}(\mathrm{dtc})_{2}\right]$ and $\left[\mathrm{Cu}(\mathrm{en})_{2}\right]^{2+}$ TPSSh and SCAN perform well, but neither method succeeds in reproducing the experimental hyperfine coupling constants quantitatively. 


\subsection{Performance of Hybrid Variants of SCAN}

The results presented above suggest that there is no one clearly superior functional that can be used reliably across all systems investigated in the present study. In terms of the role of exact exchange, the series of B3LYP, BHandHLYP, and CAM-B3LYP functionals show no definitive trend, given that the effect of the variation in exact exchange within this family of functionals depends on the compound. A methodological point that does emerge, however, is that the comparison between the mGGA functional TPSS and its hybrid variant TPSSh with $10 \%$ exact exchange is usually in favor of the latter. Therefore, admixture of exact exchange appears to be beneficial for this type of functional. This prompted us to investigate whether hybrid versions of SCAN might also offer improved performance for the property of interest. For this purpose we define two functionals, SCANh with $10 \%$ exact exchange and SCAN0 with $25 \%$ exact exchange, in direct analogy with the exact exchange percentages and the nomenclature used for the well-known TPSSh and PBE0 [69] non-empirical hybrid functionals. SCANO has been previously described by Hui and Chai [29], who similarly based the choice of $25 \%$ exact exchange on the construction of PBE0 as derived from the parent PBE GGA approximation [70,71]. We hypothesize that the lower percentage of exact exchange used in the TPSSh functional [37] might be more appropriate for a hybrid version of SCAN given the common mGGA nature of the underlying formalisms.

Compared to the values obtained with the original definition of SCAN, the exact exchange in the hybrid versions can be seen to affect all components of the hyperfine coupling. Importantly, the nature of the electronic structure that is predicted for each compound is not altered, therefore SCANh and SCANO do not afford a correct description of $\mathrm{ScO}$ and $\mathrm{TiF}_{3}$, failing in exactly the same way as the parent SCAN. The results for the two vanadium compounds demonstrate vividly that the admixture of exact exchange primarily shifts the predicted values instead of uniformly reducing the overall errors. For $\left[\mathrm{V}\left(\mathrm{H}_{2} \mathrm{O}\right)_{6}\right]^{2+}$ SCANh improves on SCAN and SCAN0 yields even better results that reproduce experiment very well. However, the corresponding results for $\left[\mathrm{VO}\left(\mathrm{H}_{2} \mathrm{O}\right)_{5}\right]^{2+}$ suggest that this agreement is fortuitous, since the increase in exact exchange in this case leads to increasing deviations from experiment.

The results for the four manganese compounds in general improve with the hybrid versions. SCAN0 is the best performing SCAN-based functional for $\mathrm{MnF}$, $\left[\mathrm{Mn}\left(\mathrm{H}_{2} \mathrm{O}\right)_{6}\right]^{2+}$, and $\mathrm{Mn}(\mathrm{CO})_{5}$, but $\mathrm{MnO}_{3}$ presents a notable exception. For this compound SCANh performs best, on par with TPSSh, but further increase of exact exchange in SCANO leads to significant deviations due to deterioration in the description of the valence orbitals. For the late transition metal complexes of the reference set $(\mathrm{Fe}, \mathrm{Ni}$, and $\mathrm{Cu}$ ) it can be concluded that the hybrid versions of SCAN either do not improve on the original or result in progressively worse agreement with experiment, an observation that holds for all copper complexes. Overall, there is no support from the present data that a global hybrid version of SCAN may be preferable for the prediction of hyperfine coupling constants.

\subsection{Basis Set Dependence of SCAN Results}

A technical point worth considering, particularly in view of the qualitative failure of SCAN for specific compounds of the present test set, is the possibility of increased sensitivity to the basis set employed in the calculation of hyperfine coupling constants. For this reason, all SCAN calculations were repeated with a different basis set that is appropriate for the target property, namely the aug-cc-pVTZ-J basis set of Hedegård, Kongsted, and Sauer [72]. The results, reported in Table 2, show no qualitative differences compared to the $\mathrm{CP}(\mathrm{PPP})$ results of Table 1. Importantly, the electronic structure obtained by SCAN for the problematic cases $\mathrm{ScO}$ and $\mathrm{TiF}_{3}$ remains invariant to the choice of basis set, and hence the Fermi contact terms for both compounds remain qualitatively incorrect. 
Table 2. Hyperfine coupling constants (in MHz) computed with SCAN and the aug-cc-pVTZ-J basis set.

\begin{tabular}{cccccccc}
\hline Compound & $A_{\mathbf{1 1}}$ & $A_{\mathbf{2 2}}$ & $A_{\mathbf{3 3}}$ & $A^{\mathrm{FC}}$ & $\boldsymbol{A}_{\mathbf{1 1}}$ SD & $\boldsymbol{A}_{\mathbf{2 2}}$ SD & $\boldsymbol{A}_{\mathbf{3 3}}$ SD \\
\hline $\mathrm{ScO}$ & -199.8 & -199.8 & -299.9 & -230.2 & 31.8 & 31.8 & -63.7 \\
$\mathrm{TiF}_{3}$ & 27.3 & 28.1 & 79.9 & 42.3 & -17.1 & -15.1 & 32.2 \\
{$\left[\mathrm{~V}\left(\mathrm{H}_{2} \mathrm{O}\right)_{6}\right]^{2+}$} & -222.2 & -222.2 & -222.5 & -214.7 & 0.1 & 0.1 & -0.2 \\
{$\left[\mathrm{VO}\left(\mathrm{H}_{2} \mathrm{O}\right)_{5}\right]^{2+}$} & -206.4 & -206.8 & -503.3 & -293.3 & 94.5 & 94.0 & -188.5 \\
$\mathrm{MnF}$ & 402.0 & 402.0 & 478.5 & 428.4 & -21.3 & -21.3 & 42.6 \\
$\mathrm{MnO}_{3}$ & 1800.2 & 1800.2 & 2081.7 & 1909.4 & -89.8 & -89.8 & 179.6 \\
{$\left[\mathrm{Mn}\left(\mathrm{H}_{2} \mathrm{O}\right)_{6}\right]^{2+}$} & -210.8 & -211.2 & -211.2 & -207.2 & 0.3 & -0.2 & -0.2 \\
$\mathrm{Mn}(\mathrm{CO})_{5}$ & -90.4 & -90.5 & 183.4 & 1.4 & -91.6 & -91.7 & 183.4 \\
{$\left[\mathrm{Fe}(\mathrm{CO})_{5}\right]^{+}$} & -17.1 & -17.1 & 34.5 & -0.4 & -17.6 & -17.6 & 35.3 \\
$\mathrm{NiH}(\mathrm{CO})_{3}$ & 53.0 & 53.0 & -100.2 & 9.2 & 54.1 & 54.2 & -108.3 \\
{$\left[\mathrm{Ni}(\mathrm{mnt})_{2}\right]^{-}$} & -6.0 & -7.2 & 59.5 & 29.3 & -31.2 & -27.2 & 58.4 \\
{$\left[\mathrm{Cu}(\mathrm{NH})_{4}\right]^{2+}$} & -63.2 & -65.1 & -721.2 & -349.2 & 247.5 & 245.5 & -493.0 \\
{$\left[\mathrm{Cu}(\mathrm{dtc})_{2}\right]$} & -118.9 & -121.6 & -544.6 & -298.5 & 157.7 & 156.1 & -313.8 \\
{$\left[\mathrm{Cu}(\mathrm{en})_{2}\right]^{2+}$} & -104.6 & -105.0 & -731.7 & -374.5 & 234.9 & 234.2 & -469.1 \\
\hline
\end{tabular}

\section{Discussion and Conclusions}

The present study was motivated by the expectation that the ability of the recently reported SCAN density functional to satisfy all known formal constraints and physical limits for a meta-GGA method might lead to better performance compared to previous density functional approximations for the theoretically and computationally challenging case of transition metal hyperfine coupling constants. SCAN and selected standard GGA, mGGA, and hybrid-mGGA functionals were compared on a selection of small $3 \mathrm{~d}$ transition metal compounds with well-characterized hyperfine coupling constants. The results suggest that the formal superiority of SCAN does not translate into a clear and uniform advantage in terms of practical applications for the property under investigation.

There are cases where SCAN performs better than BP86 GGA and the TPSS mGGA functionals, approaching or exceeding the performance of the hybrid-mGGA TPSSh despite the absence of exact exchange mixing. This is observed for both vanadium complexes of the test set, $\left[\mathrm{V}\left(\mathrm{H}_{2} \mathrm{O}\right)_{6}\right]^{2+}$ and $\left[\mathrm{VO}\left(\mathrm{H}_{2} \mathrm{O}\right)_{5}\right]^{2+}$, as well as for $\mathrm{Mn}(\mathrm{CO})_{5},\left[\mathrm{Fe}(\mathrm{CO})_{5}\right]^{+},\left[\mathrm{Cu}(\mathrm{dtc})_{2}\right]$, and $\left[\mathrm{Cu}(\mathrm{en})_{2}\right]^{2+}$, and to a lesser extent for $\mathrm{NiH}(\mathrm{CO})_{3}$. This is already an encouraging result and implies that the fundamental physics of the hyperfine coupling interaction captured by SCAN are likely improved over those of older exchange-correlation functionals. On the other hand, there are cases where SCAN underperforms significantly compared to other functionals, such as for $\left[\mathrm{Ni}(\mathrm{mnt})_{2}\right]^{-}$and $\left[\mathrm{Cu}\left(\mathrm{NH}_{3}\right)_{4}\right]^{2+}$. The most worrying situation is however encountered for two seemingly trivial examples, $\mathrm{ScO}$ and $\mathrm{TiF}_{3}$. Here SCAN fails entirely to provide meaningful results for the hyperfine coupling constants because it describes the electronic structure of these molecules in a fundamentally distinct way compared to other functionals. No other method tested here displays instances of this type of electronic structure error in the present test set, despite the occasional poor performance of any given functional for the values of the hyperfine coupling constants themselves.

The SCAN functional has inspired a series of recent studies that investigated the definition of simple hybrid as well as double-hybrid functionals using SCAN as the basic mGGA component $[28,29,31]$. As an example, SCAN0 [29], a hybrid with 25\% exact exchange, has been reported to offer improved performance for a variety of properties, including reaction energetics of late transition metal systems [31]. In this vein, we additionally studied for the spectroscopic property of interest the performance of the already described SCAN0 functional, and of the SCANh functional defined in the present study, a hybrid with $10 \%$ exact exchange. The results for the hyperfine coupling constants show that the inclusion of exact exchange shifts the predicted values by affecting core and valence spin polarization, but no clear and uniform benefit emerges: the hybrid versions may or may not perform better than SCAN in cases where SCAN is underperforming, but they also result in larger deviations in cases where SCAN already provides values that compare well with experiment. In other words, no systematic 
reduction of errors in hyperfine coupling constants can be observed upon mixing of exact exchange. This, of course, does not exclude the possibility that hybrid or double-hybrid SCAN-based functionals may systematically outperform the parent mGGA for other properties. Importantly, both hybrid versions of SCAN fail in exactly the same way as the parent functional for the problematic cases of ScO and $\mathrm{TiF}_{3}$.

The origin of these errors cannot be safely deduced from the present limited results, but the potential for this type of behavior in transition metal systems implies that SCAN needs to be evaluated more extensively in terms of its performance for the electronic structure, valence orbital description and spin-state energetics of transition metal systems before any demanding applications to spectroscopic properties. SCAN obviously offers an important platform for further methodological improvements. Additionally, with careful evaluation SCAN itself or one of its hybrid reformulations may prove useful in applications for specific transition metal ions and types of ligand environment. Nevertheless, it appears that the advanced formalism does not translate directly in improved results for hyperfine coupling interactions, suggesting that there is significant room for improvement for this property even at the level of new functional forms. Empirical benchmarking of different functionals for chemically related systems persists as the only pragmatic approach for achieving reasonably reliable predictions of transition metal hyperfine coupling constants from density functional theory.

\section{Computational Details}

All quantum chemical calculations reported in this work were performed with the ORCA package. The BP86, TPSS, TPSSh, B3LYP, BHandHLYP, CAM-B3LYP, and SCAN functionals were used as implemented in the program, while SCANh and SCAN0 were defined manually using appropriate parameters for the mixing of exact (Hartree-Fock) exchange. Geometries for all compounds were obtained from tightly converged optimizations that employed the TPSS functional with the D3 dispersion corrections [73] in conjunction with Becke-Johnson damping [74]. Geometry optimizations used the def2-TZVP basis sets [75] for all atoms and fully decontracted def2/J auxiliary basis sets [76]. Very fine integration grids (Grid6) and tight energy convergence criteria were applied throughout. A common set of geometries were used for the calculation of hyperfine coupling constants with the different functionals because calculations on selected cases showed that functional-specific geometries are not associated on average with improvements in the final predicted hyperfine coupling constants for any given method; exactly the same conclusion was reached in previous studies that investigated the performance of DFT for hyperfine coupling in transition metal complexes [10]. The core-property basis sets $\mathrm{CP}(\mathrm{PPP})$ [41] and the property-optimized aug-cc-pVTZ-J basis sets [72] were used for the transition metals in the calculation of spectroscopic parameters. These calculations employed the spin-orbit mean-field approximation and were performed with increased radial integration grids overall (IntAcc 6) and with further increased integration accuracy (SpecialGridIntAcc 12) specifically for the transition metal atoms.

Funding: This research received no external funding.

Acknowledgments: The author is grateful for the support of the Max Planck Society.

Conflicts of Interest: The author declares no conflict of interest.

\section{Appendix A}

Spin-orbit coupling is a minor contributor to the hyperfine coupling for most, but not all, compounds in this study. Its importance is more obvious for the heavier elements of the series and becomes crucial for copper compounds, for which the magnitude of $A^{\mathrm{SO}}$ is comparable to that of the other two components. Table A1 presents the spin-orbit contributions to the metal hyperfine coupling tensor for all compounds and all functionals discussed in this study. With respect to SCAN and its hybrid variants, it can be seen that the admixture of exact exchange has limited effects and clear trends emerge only for the heavier metals $(\mathrm{Ni}$ and $\mathrm{Cu}$ ), where the increase in exact exchange from SCAN 
to SCANh and SCANO is systematically accompanied with increased magnitude of the individual $A^{\mathrm{SO}}$ components.

Table A1. Spin-orbit contributions to hyperfine coupling (MHz) computed with all functionals discussed in the present work.

\begin{tabular}{|c|c|c|c|c|}
\hline Compound & Functional & $A_{11} \mathrm{SO}$ & $A_{22} \mathrm{SO}$ & $A_{33} \mathrm{SO}$ \\
\hline \multirow[t]{9}{*}{$\mathrm{ScO}$} & BP86 & -0.8 & -0.8 & -0.1 \\
\hline & TPSS & -0.8 & -0.8 & -0.1 \\
\hline & TPSSh & -0.9 & -0.9 & -0.1 \\
\hline & B3LYP & -0.9 & -0.9 & -0.1 \\
\hline & BHandHLYP & -1.0 & -1.0 & -0.2 \\
\hline & CAM-B3LYP & -0.7 & -0.7 & -0.1 \\
\hline & SCAN & -1.4 & -1.4 & -5.8 \\
\hline & SCANh & -1.4 & -1.4 & -6.0 \\
\hline & SCANO & -1.2 & -1.2 & -6.5 \\
\hline \multirow[t]{9}{*}{$\mathrm{TiF}_{3}$} & BP86 & 2.8 & 2.8 & 0.2 \\
\hline & TPSS & 2.3 & 2.3 & 0.2 \\
\hline & TPSSh & 2.5 & 2.5 & 0.2 \\
\hline & B3LYP & 3.6 & 3.6 & 0.1 \\
\hline & BHandHLYP & 5.1 & 5.1 & 0.1 \\
\hline & CAM-B3LYP & 1.0 & 1.0 & 0.1 \\
\hline & SCAN & 2.1 & 0.8 & 5.0 \\
\hline & SCANh & 0.8 & 2.2 & 5.3 \\
\hline & SCANO & 0.8 & 2.6 & 6.3 \\
\hline \multirow[t]{9}{*}[\mathrm{V}(\mathrm{H}_{2}\mathrm{O})_{6}]{$^{2+}$} & BP86 & -9.3 & -9.3 & -9.5 \\
\hline & TPSS & -8.2 & -8.2 & -8.4 \\
\hline & TPSSh & -8.5 & -8.5 & -8.7 \\
\hline & B3LYP & -10.0 & -10.0 & -10.2 \\
\hline & BHandHLYP & -10.9 & -10.9 & -11.1 \\
\hline & CAM-B3LYP & -4.0 & -4.0 & -4.0 \\
\hline & SCAN & -7.1 & -7.1 & -7.1 \\
\hline & SCANh & -7.3 & -7.3 & -7.4 \\
\hline & SCANO & -7.8 & -7.8 & -7.9 \\
\hline \multirow[t]{9}{*}[\mathrm{VO}(\mathrm{H}_{2}\mathrm{O})_{5}]{$^{2+}$} & BP86 & -7.2 & -7.4 & -25.2 \\
\hline & TPSS & -6.6 & -6.8 & -21.5 \\
\hline & TPSSh & -7.1 & -7.2 & -23.1 \\
\hline & B3LYP & -8.2 & -8.3 & -29.2 \\
\hline & BHandHLYP & -10.4 & -0.9 & -35.4 \\
\hline & CAM-B3LYP & -3.4 & -3.5 & -12.4 \\
\hline & SCAN & -7.2 & -7.1 & -19.8 \\
\hline & SCANh & -7.6 & -7.6 & -21.0 \\
\hline & SCANO & -8.4 & -8.4 & -23.2 \\
\hline \multirow[t]{9}{*}{$\mathrm{MnF}$} & BP86 & 3.8 & 3.8 & 21.5 \\
\hline & TPSS & -2.8 & -2.8 & 14.0 \\
\hline & TPSSh & -2.4 & -2.4 & 15.7 \\
\hline & B3LYP & 17.7 & 17.7 & 32.4 \\
\hline & BHandHLYP & 45.2 & 45.2 & 57.3 \\
\hline & CAM-B3LYP & -3.6 & -3.6 & 4.8 \\
\hline & SCAN & -5.1 & -5.1 & 8.7 \\
\hline & SCANh & -5.1 & -5.1 & 9.9 \\
\hline & SCANO & -4.8 & -4.8 & 12.4 \\
\hline
\end{tabular}


Table A1. Cont.

\begin{tabular}{|c|c|c|c|c|}
\hline Compound & Functional & $A_{11} \mathrm{SO}$ & $A_{22} \mathrm{SO}$ & $A_{33} \mathrm{SO}$ \\
\hline \multirow[t]{9}{*}{$\mathrm{MnO}_{3}$} & BP86 & -19.0 & -19.0 & -3.1 \\
\hline & TPSS & -17.1 & -17.0 & -3.3 \\
\hline & TPSSh & -17.8 & -17.8 & -4.8 \\
\hline & B3LYP & -19.4 & -19.4 & -7.2 \\
\hline & BHandHLYP & -13.6 & -13.6 & -0.5 \\
\hline & CAM-B3LYP & -11.4 & -11.4 & -6.0 \\
\hline & SCAN & -17.1 & -17.1 & -6.6 \\
\hline & SCANh & -18.2 & -18.2 & -9.5 \\
\hline & SCANO & -19.8 & -19.8 & -14.6 \\
\hline \multirow[t]{9}{*}[\mathrm{Mn}(\mathrm{H}_{2}\mathrm{O})_{6}]{$^{2+}$} & BP86 & -2.9 & -3.0 & -3.0 \\
\hline & TPSS & -2.9 & -2.9 & -2.9 \\
\hline & TPSSh & -2.8 & -2.8 & -2.8 \\
\hline & B3LYP & -2.6 & -2.6 & -2.6 \\
\hline & BHandHLYP & -2.1 & -2.1 & -2.1 \\
\hline & CAM-B3LYP & -2.3 & -2.3 & -2.3 \\
\hline & SCAN & -3.0 & -3.0 & -3.0 \\
\hline & SCANh & -2.8 & -2.8 & -2.8 \\
\hline & SCANO & -2.6 & -2.6 & -2.6 \\
\hline \multirow[t]{9}{*}{$\mathrm{Mn}(\mathrm{CO})_{5}$} & BP86 & 3.0 & 3.0 & -1.3 \\
\hline & TPSS & 2.1 & 2.1 & -1.6 \\
\hline & TPSSh & 2.1 & 2.1 & -2.0 \\
\hline & B3LYP & 3.6 & 3.6 & -2.2 \\
\hline & BHandHLYP & 1.6 & 1.6 & -4.7 \\
\hline & CAM-B3LYP & -1.8 & -1.8 & -1.2 \\
\hline & SCAN & 1.0 & 1.0 & -1.1 \\
\hline & SCANh & 1.0 & 1.0 & -1.3 \\
\hline & SCANO & 0.7 & 0.7 & -1.9 \\
\hline \multirow[t]{9}{*}[\mathrm{Fe}(\mathrm{CO})_{5}]{$^{+}$} & BP86 & 2.2 & 2.2 & -0.3 \\
\hline & TPSS & 1.8 & 1.8 & -0.3 \\
\hline & TPSSh & 2.2 & 2.2 & -0.4 \\
\hline & B3LYP & 3.2 & 3.2 & -0.5 \\
\hline & BHandHLYP & 4.6 & 4.6 & -0.9 \\
\hline & CAM-B3LYP & 0.5 & 0.5 & -0.3 \\
\hline & SCAN & 1.1 & 1.1 & -0.3 \\
\hline & SCANh & 1.3 & 1.3 & -0.4 \\
\hline & SCANO & 1.7 & 1.7 & -0.6 \\
\hline \multirow[t]{9}{*}{$\mathrm{NiH}(\mathrm{CO})_{3}$} & BP86 & -12.2 & -12.2 & -0.6 \\
\hline & TPSS & -12.2 & -12.2 & -0.8 \\
\hline & TPSSh & -16.6 & -16.6 & -1.5 \\
\hline & B3LYP & -23.5 & -23.5 & -2.3 \\
\hline & BHandHLYP & -56.8 & -56.8 & -8.6 \\
\hline & CAM-B3LYP & -7.5 & -7.5 & -1.0 \\
\hline & SCAN & -11.3 & -11.3 & -1.2 \\
\hline & SCANh & -14.9 & -14.9 & -2.1 \\
\hline & SCANO & -22.5 & -22.5 & -4.2 \\
\hline \multirow[t]{9}{*}[\mathrm{Ni}(\mathrm{mnt})_{2}]{$^{-}$} & BP86 & -4.0 & -10.0 & -32.8 \\
\hline & TPSS & -3.9 & -10.3 & -32.4 \\
\hline & TPSSh & -13.6 & -5.9 & -38.9 \\
\hline & B3LYP & -15.9 & -9.1 & -43.9 \\
\hline & BHandHLYP & -5.2 & -22.1 & -16.5 \\
\hline & CAM-B3LYP & -4.3 & -3.0 & -13.0 \\
\hline & SCAN & -9.9 & -4.2 & -29.6 \\
\hline & SCANh & -12.8 & -6.1 & -35.2 \\
\hline & SCANO & -17.8 & -11.5 & -44.6 \\
\hline
\end{tabular}


Table A1. Cont.

\begin{tabular}{|c|c|c|c|c|}
\hline Compound & Functional & $A_{11}$ SO & $A_{22} \mathrm{SO}$ & $A_{33} \mathrm{SO}$ \\
\hline \multirow[t]{9}{*}[\mathrm{Cu}(\mathrm{NH}_{3})_{4}]{$^{2+}$} & BP86 & 47.0 & 47.0 & 160.4 \\
\hline & TPSS & 45.6 & 45.6 & 150.3 \\
\hline & TPSSh & 53.1 & 53.1 & 174.6 \\
\hline & B3LYP & 64.8 & 64.8 & 221.4 \\
\hline & BHandHLYP & 97.2 & 97.3 & 345.7 \\
\hline & CAM-B3LYP & 23.9 & 23.9 & 87.5 \\
\hline & SCAN & 37.8 & 37.9 & 119.5 \\
\hline & SCANh & 42.8 & 42.9 & 135.0 \\
\hline & SCAN0 & 51.7 & 51.8 & 164.0 \\
\hline \multirow[t]{9}{*}[\mathrm{Cu}(\mathrm{dtc})_{2}]{} & BP86 & 20.8 & 19.4 & 68.3 \\
\hline & TPSS & 21.7 & 20.6 & 69.8 \\
\hline & TPSSh & 25.3 & 24.1 & 82.6 \\
\hline & B3LYP & 28.3 & 26.6 & 96.7 \\
\hline & BHandHLYP & 49.9 & 48.8 & 174.9 \\
\hline & CAM-B3LYP & 14.5 & 13.8 & 50.0 \\
\hline & SCAN & 21.0 & 20.1 & 65.6 \\
\hline & SCANh & 24.2 & 23.2 & 76.8 \\
\hline & SCANO & 30.3 & 29.3 & 98.1 \\
\hline \multirow[t]{9}{*}[\mathrm{Cu}(\mathrm{en})_{2}]{$^{2+}$} & BP86 & 38.6 & 39.6 & 136.5 \\
\hline & TPSS & 38.1 & 39.0 & 130.7 \\
\hline & TPSSh & 44.3 & 45.5 & 152.6 \\
\hline & B3LYP & 53.0 & 54.2 & 190.2 \\
\hline & BHandHLYP & 82.2 & 82.9 & 305.6 \\
\hline & CAM-B3LYP & 22.1 & 22.5 & 80.7 \\
\hline & SCAN & 33.9 & 34.2 & 109.5 \\
\hline & SCANh & 38.4 & 39.0 & 124.6 \\
\hline & SCANO & 46.8 & 46.9 & 152.1 \\
\hline
\end{tabular}

\section{References}

1. Kaupp, M.; Bühl, M.; Malkin, V.G. Calculation of NMR and EPR Parameters: Theory and Applications; Wiley-VCH: Weinheim, Germany, 2004; p. 603.

2. Mardirossian, N.; Head-Gordon, M. Thirty Years of Density Functional Theory in Computational Chemistry: An Overview and Extensive Assessment of 200 Density Functionals. Mol. Phys. 2017, 115, 2315-2372. [CrossRef]

3. Becke, A.D. Perspective: Fifty years of density-functional theory in chemical physics. J. Chem. Phys. 2014, 140, 18A301. [CrossRef] [PubMed]

4. Neese, F. Prediction of Molecular Properties and Molecular Spectroscopy with Density Functional Theory: From Fundamental Theory to Exchange-Coupling. Coord. Chem. Rev. 2009, 253, 526-563. [CrossRef]

5. Orio, M.; Pantazis, D.A.; Neese, F. Density Functional Theory. Photosynth. Res. 2009, 102, 443-453. [CrossRef]

6. Neese, F. High-Level Spectroscopy, Quantum Chemistry, and Catalysis: Not just a Passing Fad. Angew. Chem. Int. Ed. 2017, 56, 11003-11010. [CrossRef]

7. Munzarová, M.; Kaupp, M. A critical validation of density functional and coupled-cluster approaches for the calculation of EPR hyperfine coupling constants in transition metal complexes. J. Phys. Chem. A 1999, 103, 9966-9983. [CrossRef]

8. Munzarová, M.L.; Kubáček, P.; Kaupp, M. Mechanisms of EPR Hyperfine Coupling in Transition Metal Complexes. J. Am. Chem. Soc. 2000, 122, 11900-11913. [CrossRef]

9. Neese, F. Metal and ligand hyperfine couplings in transition metal complexes: The effect of spin-orbit coupling as studied by coupled perturbed Kohn-Sham theory. J. Chem. Phys. 2003, 118, 3939-3948. [CrossRef]

10. Kossmann, S.; Kirchner, B.; Neese, F. Performance of Modern Density Functional Theory for the Prediction of Hyperfine Structure: Meta-GGA and Double Hybrid Functionals. Mol. Phys. 2007, 105, 2049-2071. [CrossRef] 
11. Hedegård, E.D.; Kongsted, J.; Sauer, S.P.A. Improving the calculation of electron paramagnetic resonance hyperfine coupling tensors for d-block metals. Phys. Chem. Chem. Phys. 2012, 14, 10669-10676. [CrossRef]

12. Hedegård, E.D.; Kongsted, J.; Sauer, S.P.A. Validating and Analyzing EPR Hyperfine Coupling Constants with Density Functional Theory. J. Chem. Theory Comput. 2013, 9, 2380-2388. [CrossRef]

13. Schattenberg, C.J.; Maier, T.M.; Kaupp, M. Lessons from the Spin-Polarization/Spin-Contamination Dilemma of Transition-Metal Hyperfine Couplings for the Construction of Exchange-Correlation Functionals. J. Chem. Theory Comput. 2018, 14, 5653-5672. [CrossRef]

14. Sciortino, G.; Lubinu, G.; Maréchal, J.-D.; Garribba, E. DFT Protocol for EPR Prediction of Paramagnetic $\mathrm{Cu}(\mathrm{II})$ Complexes and Application to Protein Binding Sites. Magnetochemistry 2018, 4, 55. [CrossRef]

15. Heß, B.A.; Marian, C.M.; Wahlgren, U.; Gropen, O. A mean-field spin-orbit method applicable to correlated wavefunctions. Chem. Phys. Lett. 1996, 251, 365-371. [CrossRef]

16. Pantazis, D.A.; Orio, M.; Petrenko, T.; Zein, S.; Bill, E.; Lubitz, W.; Messinger, J.; Neese, F. A New Quantum Chemical Approach to the Magnetic Properties of Oligonuclear Transition-Metal Complexes: Application to a Model for the Tetranuclear Manganese Cluster of Photosystem II. Chem. Eur. J. 2009, 15, 5108-5123. [CrossRef]

17. Schraut, J.; Arbuznikov, A.V.; Schinzel, S.; Kaupp, M. Computation of Hyperfine Tensors for Dinuclear $\mathrm{Mn}^{\mathrm{III}} \mathrm{Mn}^{\mathrm{IV}}$ Complexes by Broken-Symmetry Approaches: Anisotropy Transfer Induced by Local Zero-Field Splitting. ChemPhysChem 2011, 12, 3170-3179. [CrossRef]

18. Mehlich, C.; van Wüllen, C. Broken Symmetry Approach to Magnetic Properties of Oligonuclear Transition-Metal Complexes: Application to Hyperfine Tensors of Mixed-Valence Manganese Compounds. J. Phys. Chem. C 2018, 123, 7717-7730. [CrossRef]

19. Burke, K. Perspective on density functional theory. J. Chem. Phys. 2012, 136, 150901. [CrossRef]

20. Peverati, R.; Truhlar, D.G. Quest for a universal density functional: The accuracy of density functionals across a broad spectrum of databases in chemistry and physics. Philos. Trans. R. Soc. A 2014, 372, 20120476. [CrossRef]

21. Yu, H.S.; Li, S.L.; Truhlar, D.G. Perspective: Kohn-Sham density functional theory descending a staircase. J. Chem. Phys. 2016, 145, 130901. [CrossRef]

22. Sun, J.; Ruzsinszky, A.; Perdew, J.P. Strongly Constrained and Appropriately Normed Semilocal Density Functional. Phys. Rev. Lett. 2015, 115, 36402. [CrossRef] [PubMed]

23. Brandenburg, J.G.; Bates, J.E.; Sun, J.; Perdew, J.P. Benchmark tests of a strongly constrained semilocal functional with a long-range dispersion correction. Phys. Rev. B 2016, 94, 115144. [CrossRef]

24. Sun, J.; Remsing, R.C.; Zhang, Y.; Sun, Z.; Ruzsinszky, A.; Peng, H.; Yang, Z.; Paul, A.; Waghmare, U.; Wu, X.; et al. Accurate first-principles structures and energies of diversely bonded systems from an efficient density functional. Nat. Chem. 2016, 8, 831. [CrossRef] [PubMed]

25. Goerigk, L.; Hansen, A.; Bauer, C.; Ehrlich, S.; Najibi, A.; Grimme, S. A Look at the Density Functional Theory Zoo with the Advanced GMTKN55 Database for General Main Group Thermochemistry, Kinetics and Noncovalent Interactions. Phys. Chem. Chem. Phys. 2017, 19, 32184-32215. [CrossRef] [PubMed]

26. Chen, M.; Ko, H.-Y.; Remsing, R.C.; Calegari Andrade, M.F.; Santra, B.; Sun, Z.; Selloni, A.; Car, R.; Klein, M.L.; Perdew, J.P.; et al. Ab initio theory and modeling of water. Proc. Natl. Acad. Sci. USA 2017, 114, 10846-10851. [CrossRef] [PubMed]

27. Ekholm, M.; Gambino, D.; Jönsson, H.J.M.; Tasnádi, F.; Alling, B.; Abrikosov, I.A. Assessing the SCAN functional for itinerant electron ferromagnets. Phys. Rev. B 2018, 98, 94413. [CrossRef]

28. Mezei, P.D.; Csonka, G.I.; Kállay, M. Simple Modifications of the SCAN Meta-Generalized Gradient Approximation Functional. J. Chem. Theory Comput. 2018, 14, 2469-2479. [CrossRef]

29. Hui, K.; Chai, J.-D. SCAN-based hybrid and double-hybrid density functionals from models without fitted parameters. J. Chem. Phys. 2016, 144, 44114. [CrossRef]

30. Pantazis, D.A. Assessment of Double-Hybrid Density Functional Theory for Magnetic Exchange Coupling in Manganese Complexes. Inorganics 2019, 7, 57. [CrossRef]

31. Modrzejewski, M.; Chalasinski, G.; Szczesniak, M.M. Assessment of Newest Meta-GGA Hybrids for Late Transition Metal Reactivity: Fractional Charge and Fractional Spin Perspective. J. Phys. Chem. C 2019, 123, 8047-8056. [CrossRef] 
32. Sandhoefer, B.; Kossmann, S.; Neese, F. Derivation and assessment of relativistic hyperfine-coupling tensors on the basis of orbital-optimized second-order Møller-Plesset perturbation theory and the second-order Douglas-Kroll-Hess transformation. J. Chem. Phys. 2013, 138, 104102. [CrossRef] [PubMed]

33. Neese, F. Software Update: The ORCA Program System, Version 4.0. WIREs Comput. Mol. Sci. 2018, 8, e1327. [CrossRef]

34. Becke, A.D. Density-Functional Exchange-Energy Approximation with Correct Asymptotic-Behavior. Phys. Rev. A 1988, 38, 3098-3100. [CrossRef]

35. Perdew, J.P. Density-Functional Approximation for the Correlation-Energy of the Inhomogeneous Electron-Gas. Phys. Rev. B 1986, 33, 8822-8824. [CrossRef] [PubMed]

36. Tao, J.; Perdew, J.P.; Staroverov, V.N.; Scuseria, G.E. Climbing the Density Functional Ladder: Nonempirical Meta-Generalized Gradient Approximation Designed for Molecules and Solids. Phys. Rev. Lett. 2003, 91, 146401. [CrossRef] [PubMed]

37. Staroverov, V.N.; Scuseria, G.E.; Tao, J.; Perdew, J.P. Comparative Assessment of a New Nonempirical Density Functional: Molecules and Hydrogen-Bonded Complexes. J. Chem. Phys. 2003, 119, 12129-12137. [CrossRef]

38. Becke, A.D. Density-Functional Thermochemistry. III. The Role Of Exact Exchange. J. Chem. Phys. 1993, 98, 5648-5652. [CrossRef]

39. Lee, C.; Yang, W.; Parr, R.G. Development of the Colle-Salvetti Correlation-Energy Formula Into a Functional of the Electron-Density. Phys. Rev. B 1988, 37, 785-789. [CrossRef]

40. Yanai, T.; Tew, D.P.; Handy, N.C. A new hybrid exchange-correlation functional using the Coulomb-attenuating method (CAM-B3LYP). Chem. Phys. Lett. 2004, 393, 51-57. [CrossRef]

41. Neese, F. Prediction and Interpretation of Isomer Shifts in ${ }^{57}$ Fe Mössbauer Spectra by Density Functional Theory. Inorg. Chim. Acta 2002, 337, 181-192. [CrossRef]

42. Childs, W.J.; Steimle, T.C. A molecular-beam-optical and radio frequency-optical double-resonance study of the $A^{2} \Pi_{r}-X^{2} \Sigma^{+}$band system of scandium monoxide. J. Chem. Phys. 1988, 88, 6168-6174. [CrossRef]

43. De Vore, T.C.; Weltner, W. Titanium difluoride and titanium trifluoride molecules: Electron spin resonance spectra in rare-gas matrices at 4 K. J. Am. Chem. Soc. 1977, 99, 4700-4703. [CrossRef]

44. McGarvey, B. Transition Metal Chemistry; Carlin, R.L., Ed.; Dekker: New York, NY, USA, 1966; Volume 3, pp. 89-201.

45. Grant, C.V.; Cope, W.; Ball, J.A.; Maresch, G.G.; Gaffney, B.J.; Fink, W.; Britt, R.D. Electronic Structure of the Aqueous Vanadyl Ion Probed by 9 and 94 GHz EPR and Pulsed ENDOR Spectroscopies and Density Functional Theory Calculations. J. Phys. Chem. B 1999, 103, 10627-10631. [CrossRef] [PubMed]

46. DeVore, T.C.; Van Zee, R.J.; Weltner, W., Jr. High spin molecules: ESR of MnF and $\mathrm{MnF}_{2}$ at $4^{\circ}$ K. J. Chem. Phys. 1978, 68, 3522-3527. [CrossRef]

47. Ferrante, R.F.; Wilkerson, J.L.; Graham, W.R.M.; Weltner, W. ESR spectra of the $\mathrm{MnO}, \mathrm{MnO}_{2}, \mathrm{MnO}_{3}, \mathrm{and} \mathrm{MnO}_{4}$ molecules at $4^{\circ}$ K. J. Chem. Phys. 1977, 67, 5904-5913. [CrossRef]

48. Upreti, G.C. Study of the intensities and positions of allowed and forbidden hyperfine transitions in the EPR of $\mathrm{Mn}^{2+}$ doped in single crystals of $\mathrm{Cd}\left(\mathrm{CH}_{3} \mathrm{COO}\right)_{2} \cdot 3 \mathrm{H} 2 \mathrm{O}$. J. Magn. Reson. 1974, 13, 336-347. [CrossRef]

49. Howard, J.A.; Morton, J.R.; Preston, K.F. The EPR spectrum of Mn(CO) 5 . Chem. Phys. Lett. 1981, 83, $226-228$. [CrossRef]

50. Lionel, T.; Morton, J.R.; Preston, K.F. The EPR spectrum of a single crystal of chromium hexacarbonyl doped with $\mathrm{Fe}(\mathrm{CO})_{5}$. J. Chem. Phys. 1982, 76, 234-239. [CrossRef]

51. Morton, J.R.; Preston, K.F. An ESR study at $4 \mathrm{~K}$ of the reaction between $\mathrm{H}$ and Ni(CO) $)_{4}$. J. Chem. Phys. 1984, 81, 5775-5778. [CrossRef]

52. Schmitt, R.D.; Maki, A.H. Electronic ground state of bis (maleonitrile-dithiolene)nickel monoanion. Sulfur-33 hyperfine interaction. J. Am. Chem. Soc. 1968, 90, 2288-2292. [CrossRef]

53. Scholl, H.J.; Hüttermann, J. ESR and ENDOR of copper(II) complexes with nitrogen donors: Probing parameters for prosthetic group modeling of superoxide dismutase. J. Phys. Chem. 1992, 96, 9684-9691. [CrossRef]

54. Keijzers, C.P.; Snaathorst, D. Multi-centre contributions to the anisotropic hyperfine interactions in the Cu(II) bis(dithiocarbamate) complex. Proton hyperfine couplings. Chem. Phys. Lett. 1980, 69, 348-353. [CrossRef]

55. Carter, E.; Hazeland, E.L.; Murphy, D.M.; Ward, B.D. Structure, EPR/ENDOR and DFT characterisation of a $\left[\mathrm{Cu}^{\mathrm{II}}(\mathrm{en})_{2}\right](\mathrm{OTf})_{2}$ complex. Dalton Trans. 2013, 42, 15088-15096. [CrossRef] [PubMed] 
56. Randall, D.W.; Sturgeon, B.E.; Ball, J.A.; Lorigan, G.A.; Chan, M.K.; Klein, M.P.; Armstrong, W.H.; Britt, R.D. ${ }^{55} \mathrm{Mn}$ ESE-ENDOR of a Mixed Valence Mn(III)Mn(IV) Complex: Comparison with the Mn Cluster of the Photosynthetic Oxygen-Evolving Complex. J. Am. Chem. Soc. 1995, 117, 11780-11789. [CrossRef]

57. Peloquin, J.M.; Campbell, K.A.; Britt, R.D. ${ }^{55} \mathrm{Mn}$ Pulsed ENDOR Demonstrates That the Photosystem II "Split" EPR Signal Arises from a Magnetically-Coupled Mangano-Tyrosyl Complex. J. Am. Chem. Soc. 1998, 120, 6840-6841. [CrossRef]

58. Peloquin, J.M.; Campbell, K.A.; Randall, D.W.; Evanchik, M.A.; Pecoraro, V.L.; Armstrong, W.H.; Britt, R.D. ${ }^{55} \mathrm{Mn}$ ENDOR of the $\mathrm{S}_{2}$-State Multiline EPR Signal of Photosystem II: Implications on the Structure of the Tetranuclear Mn Cluster. J. Am. Chem. Soc. 2000, 122, 10926-10942. [CrossRef]

59. Kulik, L.V.; Epel, B.; Lubitz, W.; Messinger, J. ${ }^{55} \mathrm{Mn}$ Pulse ENDOR at $34 \mathrm{GHz}$ of the $\mathrm{S}_{0}$ and $\mathrm{S}_{2}$ States of the Oxygen-Evolving Complex in Photosystem II. J. Am. Chem. Soc. 2005, 127, 2392-2393. [CrossRef] [PubMed]

60. Cox, N.; Ames, W.; Epel, B.; Kulik, L.V.; Rapatskiy, L.; Neese, F.; Messinger, J.; Wieghardt, K.; Lubitz, W. Electronic Structure of a Weakly Antiferromagnetically Coupled $\mathrm{Mn}^{\mathrm{II}} \mathrm{Mn}^{\mathrm{III}}$ Model Relevant to Manganese Proteins: A Combined EPR, ${ }^{55} \mathrm{Mn}-\mathrm{ENDOR}$, and DFT Study. Inorg. Chem. 2011, 50, 8238-8251. [CrossRef]

61. Cox, N.; Rapatskiy, L.; Su, J.-H.; Pantazis, D.A.; Sugiura, M.; Kulik, L.; Dorlet, P.; Rutherford, A.W.; Neese, F.; Boussac, A.; et al. Effect of $\mathrm{Ca}^{2+} / \mathrm{Sr}^{2+}$ substitution on the electronic structure of the oxygen-evolving complex of photosystem II: A combined multifrequency EPR, ${ }^{55} \mathrm{Mn}-\mathrm{ENDOR}$, and DFT study of the $\mathrm{S}_{2}$ state. J. Am. Chem. Soc. 2011, 133, 3635-3648. [CrossRef]

62. Mukherjee, S.; Stull, J.A.; Yano, J.; Stamatatos, T.C.; Pringouri, K.; Stich, T.A.; Abboud, K.A.; Britt, R.D.; Yachandra, V.K.; Christou, G. Synthetic model of the asymmetric $\left[\mathrm{Mn}_{3} \mathrm{CaO}_{4}\right]$ cubane core of the oxygen-evolving complex of photosystem II. Proc. Natl. Acad. Sci. USA 2012, 109, 2257-2262. [CrossRef]

63. Shafaat, H.S.; Griese, J.J.; Pantazis, D.A.; Roos, K.; Andersson, C.S.; Popović-Bijelić, A.; Gräslund, A.; Siegbahn, P.E.M.; Neese, F.; Lubitz, W.; et al. Electronic Structural Flexibility of Heterobimetallic Mn/Fe Cofactors: R2lox and R2c Proteins. J. Am. Chem. Soc. 2014, 136, 13399-13409. [CrossRef] [PubMed]

64. Orio, M.; Pantazis, D.A.; Petrenko, T.; Neese, F. Magnetic and Spectroscopic Properties of Mixed Valence Manganese(III, IV) Dimers: A Systematic Study Using Broken Symmetry Density Functional Theory. Inorg. Chem. 2009, 48, 7251-7260. [CrossRef] [PubMed]

65. Pantazis, D.A.; Orio, M.; Petrenko, T.; Zein, S.; Lubitz, W.; Messinger, J.; Neese, F. Structure of the oxygen-evolving complex of photosystem II: Information on the $S_{2}$ state through quantum chemical calculation of its magnetic properties. Phys. Chem. Chem. Phys. 2009, 11, 6788-6798. [CrossRef] [PubMed]

66. Schinzel, S.; Kaupp, M. Validation of Broken-Symmetry Density Functional Methods for the Calculation of Electron Paramagnetic Resonance Parameters of Dinuclear Mixed-Valence $\mathrm{Mn}^{\mathrm{IV}} \mathrm{Mn}^{\mathrm{III}}$ Complexes. Can. J. Chem. 2009, 87, 1521-1539. [CrossRef]

67. Schinzel, S.; Schraut, J.; Arbuznikov, A.V.; Siegbahn, P.E.M.; Kaupp, M. Density Functional Calculations of ${ }^{55} \mathrm{Mn},{ }^{14} \mathrm{~N}$ and ${ }^{13} \mathrm{C}$ Electron Paramagnetic Resonance Parameters Support an Energetically Feasible Model System for the $\mathrm{S}_{2}$ State of the Oxygen-Evolving Complex of Photosystem II. Chem.-A Eur. J. 2010, 16, 10424-10438. [CrossRef]

68. Krewald, V.; Neese, F.; Pantazis, D.A. On the Magnetic and Spectroscopic Properties of High-Valent $\mathrm{Mn}_{3} \mathrm{CaO}_{4}$ Cubanes as Structural Units of Natural and Artificial Water Oxidizing Catalysts. J. Am. Chem. Soc. 2013, 135, 5726-5739. [CrossRef]

69. Adamo, C.; Barone, V. Toward Reliable Density Functional Methods Without Adjustable Parameters: The PBE0 Model. J. Chem. Phys. 1999, 110, 6158-6170. [CrossRef]

70. Perdew, J.P.; Burke, K.; Ernzerhof, M. Generalized gradient approximation made simple. Phys. Rev. Lett. 1996, 77, 3865-3868. [CrossRef]

71. Perdew, J.P.; Burke, K.; Ernzerhof, M. Generalized Gradient Approximation Made Simple. Phys. Rev. Lett. 1997, 77, 3865, Erratum in Phys. Rev. Lett. 1997, 78, 1396. [CrossRef]

72. Hedegård, E.D.; Kongsted, J.; Sauer, S.P.A. Optimized Basis Sets for Calculation of Electron Paramagnetic Resonance Hyperfine Coupling Constants: Aug-cc-pVTZ-J for the 3d Atoms Sc-Zn. J. Chem. Theory Comput. 2011, 7, 4077-4087. [CrossRef]

73. Grimme, S.; Antony, J.; Ehrlich, S.; Krieg, H. A Consistent and Accurate ab initio Parametrization of Density Functional Dispersion Correction (DFT-D) for the 94 Elements H-Pu. J. Chem. Phys. 2010, 132, 154104. [CrossRef] [PubMed] 
74. Grimme, S.; Ehrlich, S.; Goerigk, L. Effect of the Damping Function in Dispersion Corrected Density Functional Theory. J. Comput. Chem. 2011, 32, 1456-1465. [CrossRef] [PubMed]

75. Weigend, F.; Ahlrichs, R. Balanced Basis Sets of Split Valence, Triple Zeta Valence and Quadruple Zeta Valence Quality for H to Rn: Design and Assessment of Accuracy. Phys. Chem. Chem. Phys. 2005, 7, 3297-3305. [CrossRef] [PubMed]

76. Weigend, F. Accurate Coulomb-Fitting Basis Sets for H to Rn. Phys. Chem. Chem. Phys. 2006, 8, 1057-1065. [CrossRef]

(C) 2019 by the author. Licensee MDPI, Basel, Switzerland. This article is an open access article distributed under the terms and conditions of the Creative Commons Attribution (CC BY) license (http://creativecommons.org/licenses/by/4.0/). 